\title{
The Future in Diagnosis and Staging of Lung Cancer: Introduction
}

\author{
Johan Vansteenkiste \\ Respiratory Oncology (Pulmonology) and Leuven Lung Cancer Group, University Hospital Gasthuisberg, \\ Leuven, Belgium
}

Lung cancer is the major cause of cancer-related death in Europe, accounting for about 20\% of total cancer deaths. In men, lung cancer is responsible for about $28 \%$ of cancer deaths, far more than the second in importance, prostate cancer, with approximately $12 \%$. In women, breast cancer is the leading cause of death from cancer at $18 \%$, with lung cancer being the second cause in many countries, accounting for about $10 \%$ of cancer deaths.

Despite significant advances in various modalities of treatment, the overall prognosis remains limited because of the aggressive nature of this neoplasm and the late stage of presentation in many patients.

The most effective way to reduce lung cancer mortality in the long-term is without doubt the reduction of smoking habits. In many European countries, campaigns and public health measures have been developed to combat smoking as one of the major killers in society.

Another possibly effective means of reducing lung cancer mortality might be the successful identification of patients with early cancers in high-risk populations. The use of low-dose spiral computed tomography (CT) in lung cancer screening and the use of new molecular markers has brought realistic hope that these novel approaches may do better that the historical experience with chest $\mathrm{X}$-ray and simple sputum cytology [1]. The first contribution to this series by Pastorino et al. will focus on the current knowledge of lung cancer screening and the need for validation of this concept in randomized controlled trials.

\section{KARGER \\ Fax +4161306 1234 E-Mail karger@karger.ch} www.karger.com
(C) 2006 S. Karger AG, Base 0025-7931/06/0731-0003\$23.50/0

Accessible online at: www.karger.com/res
In patients with lung cancer beyond truly early stages, most of the progress has been made by multimodality treatment approaches, combining modern chemotherapy, surgery and radiotherapy in different indications and sequences $[2,3]$. Accurate locoregional (and overall) staging is of critical importance for the optimal indication of these often demanding multimodality treatments. Lack of appropriate staging leads to inadequate therapeutic trials and a waste of therapeutic efforts both for the patients and the caregivers. Fortunately, the last decade was also characterized by major steps forward in the staging process as well.

$\mathrm{CT}$ of the chest and upper abdomen has gained a central role in this staging process since decades. The significant technological advances in recent years will be reviewed in a second contribution by Laurent et al. With their excellent anatomic performance, modern multislice spiral CT scanners give valuable details to assess tumor spread and to direct ensuing invasive procedures. Rapid 3D reformations enable imaging in all planes, thereby obviating most of the historical indications for magnetic resonance imaging.

The third review by Fischer et al. will highlight how positron emission tomography (PET) has improved overall lung cancer staging. PET complements traditional imaging in a very substantial way because it is able to study metabolic processes in tissues, thereby allowing accurate characterization of the primary tumor, evaluation of locoregional lymph node spread, and assessment for distant 
metastases in one single noninvasive test. Ongoing studies are examining how PET-CT fusion scanners may further improve the staging capabilities of cancer imaging.

Apart from cancer imaging, substantial improvement has taken place in the different techniques that assess the disease by looking at cytology and tissue samples as well. Bronchoscopy, obligatory in most patients with suspected lung cancer, has extended its possibilities far beyond a simple biopsy of an endobronchially visible lesion. It permits an accurate evaluation of the endobronchial extension of the primary tumor. Herth et al. will review how the possibilities for locoregional lymph node staging have evolved from blind transbronchial needle aspiration to transbronchial imaging beyond the mucosa with endoscopic ultrasonography, either by the transesophageal or transbronchial route. The latest bronchoscopic development, real-time endobronchial ultrasonography permits lymph node sampling under direct ultrasound visualization.

Mediastinoscopy has been the gold standard for locoregional lymph node staging in patients with lung cancer for many decades. Goldstraw et al. will argue that invasive surgical staging will continue to play a role in quite a few patients in the era of rapidly evolving nonin- vasive and minimally invasive staging techniques. The new techniques allow better indication and guidance of the surgical procedures. Careful intra-operative staging by the surgeon yields the most accurate TNM and is of major importance to optimize the selection of patients for adjuvant therapies.

Finally, the use of antibodies and other molecular techniques have brought the accuracy of the pathologist in the detection and characterization of cancer cells in preoperatively and intra-operatively sampled tissues far beyond what was possible with simple light microscopy. Passlick et al. will summarize how this has impacted on and may further improve lung cancer staging.

The availability of various highly accurate diagnostic and staging tools has clearly improved our management of patients with lung cancer. These often complimentary techniques are our best guide to plan the optimal treatment for each patient. In the baseline staging, some of these new tests have already been shown to improve therapeutic strategies [4]. How and when to use the different possibilities in the ever increasing modern multimodality treatments will be the next challenge that needs to be further evaluated in well designed prospective studies.

\section{References}

1 Novello S, Fava C, Borasio P, et al: Three-year findings of an early lung cancer detection feasibility study with low-dose spiral computed tomography in heavy smokers. Ann Oncol 2005; 16:1662-1666

2 Vansteenkiste JF, Schildermans RH: The future of adjuvant chemotherapy for resected non-small cell lung cancer. Expert Rev Anticancer Ther 2005;5:165-175.
3 Rowell NP, O'Rourke NP: Concurrent chemoradiotherapy in non-small cell lung cancer. Cochrane Database Syst Rev 2004;CD002140.

4 Van Tinteren H, Hoekstra OS, Smit EF, et al: Effectiveness of positron emission tomography in the preoperative assessment of patients with suspected non-small cell lung cancer: the PLUS multicentre randomised trial. Lancet 2002; 359:1388-1393. 